\title{
Simulating the performance of MaxEnTes temperature-emissivity separation algorithm
}

\author{
by *A. Barducci, *D. Guzzi, *C.Lastri, *V.Nardino, *P. Marcoionni, and *I.Pippi
}

* Istituto di Fisica Applicata "Nello Carrara", Consiglio Nazionale delle Ricerche, Via Madonna del Piano 1050019 Sesto Fiorentino, Firenze, Italy, i.pippi@ifac.cnr.it

\section{Abstract}

Temperature and emissivity separation (TES) as applied to multispectral Thermal Infrared (TIR) images of the Earth surface provides temperature and emissivity, relevant parameters for Earth observation studies. Retrieval of temperature and emissivity is an ill-posed problem that admits infinite solutions because the number of unknowns goes beyond the available measurements. In this work the Maximum Entropy Temperature and Emissivity Separation (MaxEnTes) devoted to the retrieval of both temperature and spectral emissivity of a remote target is introduced and described summarizing the physical implications of the information theory and the meaning of the information entropy.

\section{Introduction}

The use of multiband TIR detectors provides the experimental determination of the ground emitted radiance $L_{i}$ in various spectral channels. This spectral radiance can be expressed as the product of the Planck law (calculated for a target temperature T) and the emissivity $\varepsilon(\lambda)$. For an ideal channel having bandwidth $\Delta \lambda$ the radiance spectrum can be written as:

$$
L_{i}=\int_{(\Delta \lambda)_{i}} \varepsilon(\lambda) \frac{c_{1}}{\lambda^{5}} \frac{1}{\exp \left(c_{2} / \lambda T\right)-1} d \lambda
$$

where $c_{1}=2 h c^{2}, c_{2}=h c / k, k$ is the Boltzmann's constant, $h$ is the Planck's constant, and $c$ is the speed of light. Using band-integrated quantities, we can write:

$$
L_{i}=\varepsilon\left(\lambda_{i}\right) B\left(T, \lambda_{i}\right)=\varepsilon_{i} B_{i}
$$

with $\lambda_{i}$ effective wavelength, $B_{i}=B\left(T, \lambda_{i}\right)$ Planck function and $\varepsilon_{i}=\varepsilon\left(\lambda_{i}\right)$ emissivity in the $i$-th channel, $i=1, \ldots, n$. As shown in several previous papers discussing this topic, the retrieval of both temperature and emissivity is a problem admitting infinite solutions. Different approaches have been proposed for the solution of this problem [1, 2].

A large effort has been devoted to develop new methodologies of fitting, and inverse modelling able to handle cases in which the number of unknowns exceeds the number of measurements. These procedures took the collective name of Maximum Entropy formalism (MaxEnt) [3, 4].

In this paper, an advanced temperature/emissivity separation algorithm based on the MaxEnt approach is presented. The method starts from the assumption that the condition of maximum entropy is the less committal hypothesis that can be made to overcome the lack of knowledge, preventing an algebraic solution to the temperature emissivity separation problem. The general framework of MaxEnt will be depicted and the meaning of the information entropy in [3-6] will be discussed. Next, we describe in detail the developed algorithm for assessing target's temperature and emissivity, and finally, some conclusions are drawn.

\section{MaxEnt formalism}

Using the MaxEnt methodology $n$ unknowns of interest (temperature and emissivity in our case) are obtained as expectation values of corresponding random variables. The problem of inferring these unknowns from $m<n$ measurements is therefore turned into the problem of assessing the Probability Density Function (PDF) of these random variables. Such a function is deduced requiring its entropy to be maximal, subject to a set of constraints representing the available experimental outcomes.

We consider a set of possible values $\left\{x_{i}\right\}$ for a random variable $x$ having probability $p\left(x_{i}\right)$. The information entropy is defined as:

$$
H=-\sum_{i} p\left(x_{i}\right) \log p\left(x_{i}\right) ; \quad p\left(x_{i}\right) \geq 0
$$


The accepted maximum entropy PDF is constrained so the expected value $\eta_{f_{k}(x)}=E\left[f_{k}(x)\right]$ of a set of functions $f_{k}(x)$ exactly matches the available measurements $L_{k}$ :

$$
\eta_{f_{k}(x)}=\sum_{i} p\left(x_{i}\right) f_{k}\left(x_{i}\right)=L_{k}, k=1, \ldots, m
$$

and $\boldsymbol{E}[\boldsymbol{]}$ is the ensemble-average operator. Maximization of $\boldsymbol{H}$ yields the following result:

$$
p\left(x_{i}\right)=\frac{\exp \left(I_{1} f_{1}\left(x_{i}\right)+\ldots+I_{m} f_{m}\left(x_{i}\right)\right)}{Z\left(I_{1}, \ldots, I_{m}\right)}
$$

With $\boldsymbol{Z}$ the partition function and $I_{1}, \ldots, l_{m}$ the Lagrange multipliers for the minimization of $\boldsymbol{H}$, given by:

$$
\begin{aligned}
& Z\left(I_{1}, \ldots, I_{m}\right)=\sum_{i} \exp \left(\sum_{k=1}^{m} I_{k} f_{k}\left(x_{i}\right)\right) \\
& I_{k}: \frac{\partial}{\partial I_{k}} \log Z\left(I_{1}, \ldots, I_{m}\right)=\eta_{f_{k}(x)}
\end{aligned}
$$

The extension of this formalism to the case of continuous variables can be easily attained turning any summation into the corresponding integral and including a metric function $\mu(x)$ that makes the entropy definition independent of coordinate changes:

$$
H=-\int p(x) \log \frac{p(x)}{\mu(x)} d x
$$

The MaxEnt solution for this case is summarized in the following relationships:

$$
\begin{aligned}
& p(x)=\frac{\mu(x) \exp \left(I_{1} f_{1}(x)+\ldots+I_{m} f_{m}(x)\right)}{Z\left(I_{1}, \ldots, I_{m}\right)} \\
& Z\left(I_{1}, \ldots, I_{m}\right)=\int \mu(x) \exp \left(\sum_{k=1}^{m} I_{k} f_{k}(x)\right) d x
\end{aligned}
$$

The goal of this work is to estimate the expectation value of both temperature $T$ and emissivity spectrum $\varepsilon_{i}$ calculated in the generic $i$-th channels as expectation values of corresponding aleatory variables $\boldsymbol{e}_{\boldsymbol{i}}, \boldsymbol{t}$.

$$
\begin{aligned}
& E\left\{e_{k}\right\}=\int_{\Omega_{T}} \int_{\Omega_{E_{1}}} \ldots \int_{\Omega_{E_{n}}} \mu\left(e_{1}, \ldots, e_{n}, t\right) e_{k} p\left(e_{1}, \ldots, e_{n}, t\right) d e_{1}, \ldots, d e_{n} d t \\
& E\{t\}=\int_{\Omega_{T}} \int_{\Omega_{E_{1}}} \ldots \int_{\Omega_{E_{n}}} \mu\left(e_{1}, \ldots, e_{n}, t\right) t p\left(e_{1}, \ldots, e_{n}, t\right) d e_{1}, \ldots, d e_{n} d t
\end{aligned}
$$

where the metric $\mu\left(e_{1}, \ldots, e_{n}, t\right)$ defines the integration boundaries in each domain $\Omega_{T}, \Omega_{E_{1}}, \ldots, \Omega_{E_{n}}$. Such a metric is used to assign a different weight to different states of the system. In the following section it will be chosen to include physical constraints about the expected temperature and the emissivity of the target.

The corresponding emitted radiance measured in the $i$-th channel can be written as:

$$
L_{i}=L_{i}\left(\lambda_{i}, t\right)=e\left(\lambda_{i}\right) B\left(\lambda_{i}, t\right)
$$

The Lagrange multipliers are calculated using Eq. (7) i.e. by imposing the measured values $L_{i}$ to satisfy:

$$
L_{i}=E\left\{e\left(\lambda_{i}\right) B\left(\lambda_{i}, t\right)\right\}
$$

The above expression involves a product between the emissivity and an exponential function, where in general $E\left\{L_{i}\right\}=E\left\{e\left(\lambda_{i}\right) B\left(\lambda_{i}, t\right)\right\} \neq E\left\{e\left(\lambda_{i}\right)\right\}\left\{B\left(\lambda_{i}, t\right)\right\}$. There are notable difficulties in this formulation, due to the non-analytical solution of Eq. (14) and to the presence of exponential of Planck functions in Eqs. (9) and (10). Moreover, the presence of discontinuities in the multi-dimensional domain of Eq. (14) makes problematic the convergence of the numerical solution. 


\section{First order approximation}

In order to calculate the coefficients $I_{1}, \ldots, I_{m}$ the emitted radiance in the channel $L_{i}$ has been expanded in a series stopped at the first order term:

Defining:

$$
L_{i}\left(\lambda_{i}, t_{0}+\Delta t\right) \cong e_{0}\left(\lambda_{i}\right) B\left(\lambda_{i}, t_{0}\right)+\Delta e\left(\lambda_{i}\right) B\left(\lambda_{i}, t_{0}\right)+e_{0}\left(\lambda_{i}\right) \Delta B\left(\lambda_{i}, t_{0}\right)
$$

$$
\Delta B\left(\lambda_{i}, t_{0}\right)=B_{i} \frac{\exp \left(c_{2} / \lambda_{i} t_{0}\right)}{\exp \left(c_{2} / \lambda_{i} t_{0}\right)-1} \frac{c_{2}}{\lambda_{i} t_{0}^{2}} \Delta t=B_{i} \alpha_{i} \Delta t
$$

we can write:

$$
L_{i}\left(\lambda_{i}, t_{0}+\Delta t\right) \cong \tilde{L}_{i}\left(\lambda_{i}, t_{0}+\Delta t\right)=e_{0 i} B_{i}+\Delta e_{i} B_{i}+e_{0 i} B_{i} \alpha_{i} \Delta t
$$

where $\Delta t$ and $\Delta e_{i}$ are new variables and $e_{0 i}, B_{i}$ and $\alpha_{i}$ are constants fixed by the value of $t_{0}$. Within this approximation we can write:

$$
E\left\{e\left(\lambda_{i}\right) B\left(\lambda_{i}, t\right)\right\}=E\left\{e\left(\lambda_{i}\right) B\left(\lambda_{i}, t\right)\right\}=e_{0 i} B_{i}+E\left\{\Delta e_{i}\right\} B_{i}+e_{0 i} B_{i} \alpha_{i} E\{\Delta t\}
$$

The partition function may be rewritten as:

$$
Z\left(I_{1}, \ldots, I_{m}\right)=\int \ldots \int m\left(\Delta e_{1}, \ldots, \Delta e_{n}, \Delta t\right) \exp \left(\sum_{k=1}^{m} I_{k} \tilde{L}_{k}\right) d\left(\Delta e_{1}\right) \ldots d\left(\Delta e_{n}\right) d(\Delta t)
$$

where $m\left(\Delta e_{1}, \ldots, \Delta e_{n}, \Delta t\right)$ is used as a metric for defining the limits of the integration domain:

$$
m\left(\Delta e_{1}, \ldots, \Delta e_{n}, \Delta t\right)= \begin{cases}0 & \Delta e_{k} \notin\left[e_{\min }-e_{0 k}, e_{\max }-e_{0 k}\right] \wedge \Delta t \notin\left[T_{\min }-t_{0}, T_{\max }-t_{0}\right] \\ 1 & \Delta e_{k} \in\left[e_{\min }-e_{0 k}, e_{\max }-e_{0 k}\right] \vee \Delta t \in\left[T_{\min }-t_{0}, T_{\max }-t_{0}\right]\end{cases}
$$

The $I_{1}, \ldots, I_{m}$ values can be obtained by substituting $\tilde{L}_{i}$ in Eq. (7). Due to the fact that separate constraints on both temperature and emissivity don't allow the convergence of the calculation (i.e. the solution of Eq. (7),) the solution of this equation is performed numerically.

Using the probability distribution function Eq. (5), the emissivity and temperature values are finally calculated as:

$$
\begin{aligned}
& E\left\{\Delta e_{k}\right\}=\int_{T_{0}}^{T_{1}} \int_{\Delta e_{\min 1}}^{\Delta e_{\max 1}} \ldots \int_{\Delta e_{\min n}}^{\Delta e_{\max }} \Delta e_{k} p\left(\Delta e_{1}, \ldots, \Delta e_{n}, \Delta t\right) d\left(\Delta e_{1}\right), \ldots, d\left(\Delta e_{n}\right) d(\Delta t) \\
& E\{\Delta t\}=\int_{T_{0}}^{T_{1}} \int_{\Delta e_{\min 1}}^{\Delta e_{\max }} \ldots \int_{\Delta e_{\min n}}^{\Delta e_{\max }} \Delta t p\left(\Delta e_{1}, \ldots, \Delta e_{n}, \Delta t\right) d\left(\Delta e_{1}\right), \ldots, d\left(\Delta e_{n}\right) d(\Delta t)
\end{aligned}
$$

being $\Delta e_{\min k}=e_{\min }-e_{0 k}$ for $k=1, \ldots, n$ and $T_{0}=T_{\min }-t_{0}-\left(T_{\max }-T_{\min }\right) / 4, T_{1}=T_{\max }-t_{0}+\left(T_{\max }-T_{\min }\right) / 4$.

Since the integration of Eqs. (21) and (22) can been analytically performed, the computation speed results increased with respect to numerical integration allowing the application to large amount of data (i.e. spectral datacubes from remotely sensed images).

\section{Temperature Range Selection (TRS) algorithm}

In order to define the variables range to be used as metric function, realistic values for the variables have to be defined on the basis of the expected variability of temperature and emissivity of the observed target.

In particular, we assume that the observed brightness temperature in a generic channel is always lower than its real value due to the emissivity (always less than the unity). If we set $e_{\max }, e_{\min }$ as the (theoretical) maximum and minimum accepted values of emissivity, a brightness temperature spectrum can be calculated from the measured emitted radiance value $L(\lambda)$ for both the minimum and maximum emissivity value, as:

$$
\begin{array}{ll}
T_{B M_{\varepsilon=e_{\min }}}(\lambda)=B^{-1}\left(L(\lambda) / e_{\min }\right), & e_{\min }=0.8 \\
T_{B_{\varepsilon=e_{\max }}}(\lambda)=B^{-1}\left(L(\lambda) / e_{\max }\right), & e_{\max }=0.999
\end{array}
$$


being $T_{B M_{\varepsilon=e_{\min }}}\left(\lambda_{k}\right)$ the maximum theoretical brightness temperature (case of minimum emissivity for the majority of natural targets) and $T_{B_{\varepsilon}=e_{\max }}\left(\lambda_{k}\right)$ the brightness temperature for the maximum emissivity (always less than the unity) in each $\boldsymbol{K}$-th channel.

The temperature spectra $T_{B M \varepsilon=e_{\min }}\left(\lambda_{k}\right)$ and $T_{B \varepsilon=e_{\max }}\left(\lambda_{k}\right)$ force the target temperature between the minimum value of the $T_{B M_{\varepsilon=e_{\min }}}\left(\lambda_{k}\right)$ and the maximum value of $T_{B \varepsilon=e_{\max }}\left(\lambda_{k}\right)$, thus we define the admitted temperature range $T_{\min }{ }^{-}$ $T_{\max }$ as:

$$
\begin{aligned}
& T_{\text {min }}=\max \left\{T_{B \varepsilon=e_{\max }}\left(\lambda_{k}\right), t_{m}\right\}, \quad k=1, \ldots, n, \quad t_{m}=283 \mathrm{~K} \\
& T_{\text {max }}=\min \left\{T_{B M_{\varepsilon=e_{\min }}}\left(\lambda_{k}\right), t_{M}\right\} \quad k=1, \ldots, n, \quad t_{M}=323 \mathrm{~K}
\end{aligned}
$$

$t_{m}, t_{M}$ being, respectively, local and seasonal minimum and maximum values used for preventing outliers.

Using $T_{\max }, T_{\min }$, the value of temperature $t_{0}$ used for the linear approximation can be defined using as. The first guess for the emissivity in the $i$-th channel is given by $e_{0 i}=e_{0}\left(\lambda_{i}\right)=L_{i} / B\left(t_{0}\right)$ with:

$$
B_{i}=B_{i}\left(\lambda_{i}, t_{0}\right)=\frac{c_{1}}{\lambda_{i}^{5}} \frac{1}{\exp \left(c_{2} / \lambda_{i} t_{0}\right)-1}
$$

\section{MaxEnTES algorithm}

The MaxEnTES (Maximum Entropy Temperature and Emissivity Separation) algorithm is made up of two different modules: the TRS algorithm and the MaxEnt method. The entire procedure is summarized in figure 1.

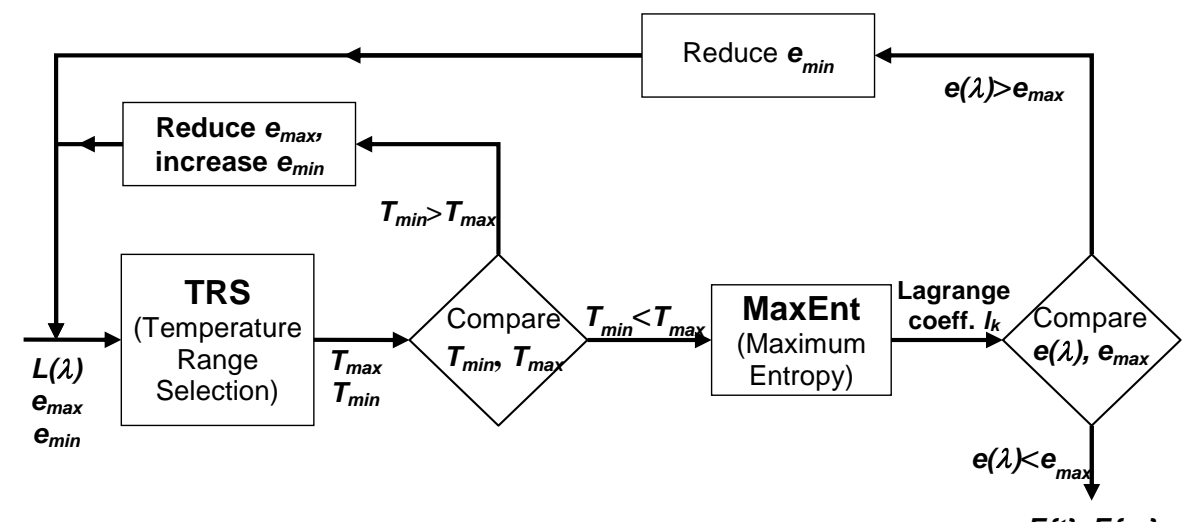

$E\{t\}, E\left\{e_{k}\right\}$

Fig. 1. Scheme of MaxEnTES (Maximum Entropy Temperature and Emissivity Separation) algorithm. The procedure is made up of two blocks: the Temperature Range Selection (TRS) and the Maximum Entropy (MaxEnt) algorithms.

First the TRS algorithm is used for an estimate of the emissivity and temperature range from the radiance direct measurement: using as input the emissivity range $e_{\max }, e_{\min }$ and the seasonal extreme temperature values $t_{m}, t_{M}$, it provides $T_{\min }, T_{\max }$. If $T_{\min }>T_{\max }$ these values are rejected and the TRS algorithm is iterated enlarging the range $e_{\max }$, $e_{\min }$ until $T_{\min } \leq T_{\max }$. At this point the $t_{0}$ value is calculated using the MaxEnt method and the first order approximation is used for determining the $I_{1}, \ldots, I_{m}$ values defining the probability distribution function that maximizes the entropy (i.e. MaxEnt approach). If the emissivity spectrum overpasses $e_{\max }$, the sequence of the TRS and MaxEnt is repeated after reducing $e_{\min }$. It is important to point out that, during the TRS algorithm, both the values $e_{\min }$, $e_{\max }$ are updated for enlarging the emissivity admitted range. On the other hand, after the MaxEnt procedure, if the emissivity spectrum overpasses $e_{\max }$, only the $e_{\min }$ value is reduced. In other words, the MaxEnTES procedure automatically enlarge the $e_{\min }, e_{\max }$ range for emissivity until it is automatically centred on the target emissivity spectrum. 


\section{Tests and results}

Measured emissivity spectra extracted from the Johns Hopkins University Spectral Library [7] have been used to test MaxEnTES procedure. Two different datasets of minerals (of geological interest) and natural/artificial targets have been selected. The emitted radiance has been simulated by setting for the target a fixed value of the temperature, equal to $300^{\circ} \mathrm{K}$, and calculating the corresponding Planck function. The radiance spectrum is represented by the product of the emissivity spectrum with the Planck function for fixed temperature value. The value in each channel (considered ideal) is obtained by spectral averaging. Results coming from simulations are reported in figure 2 .

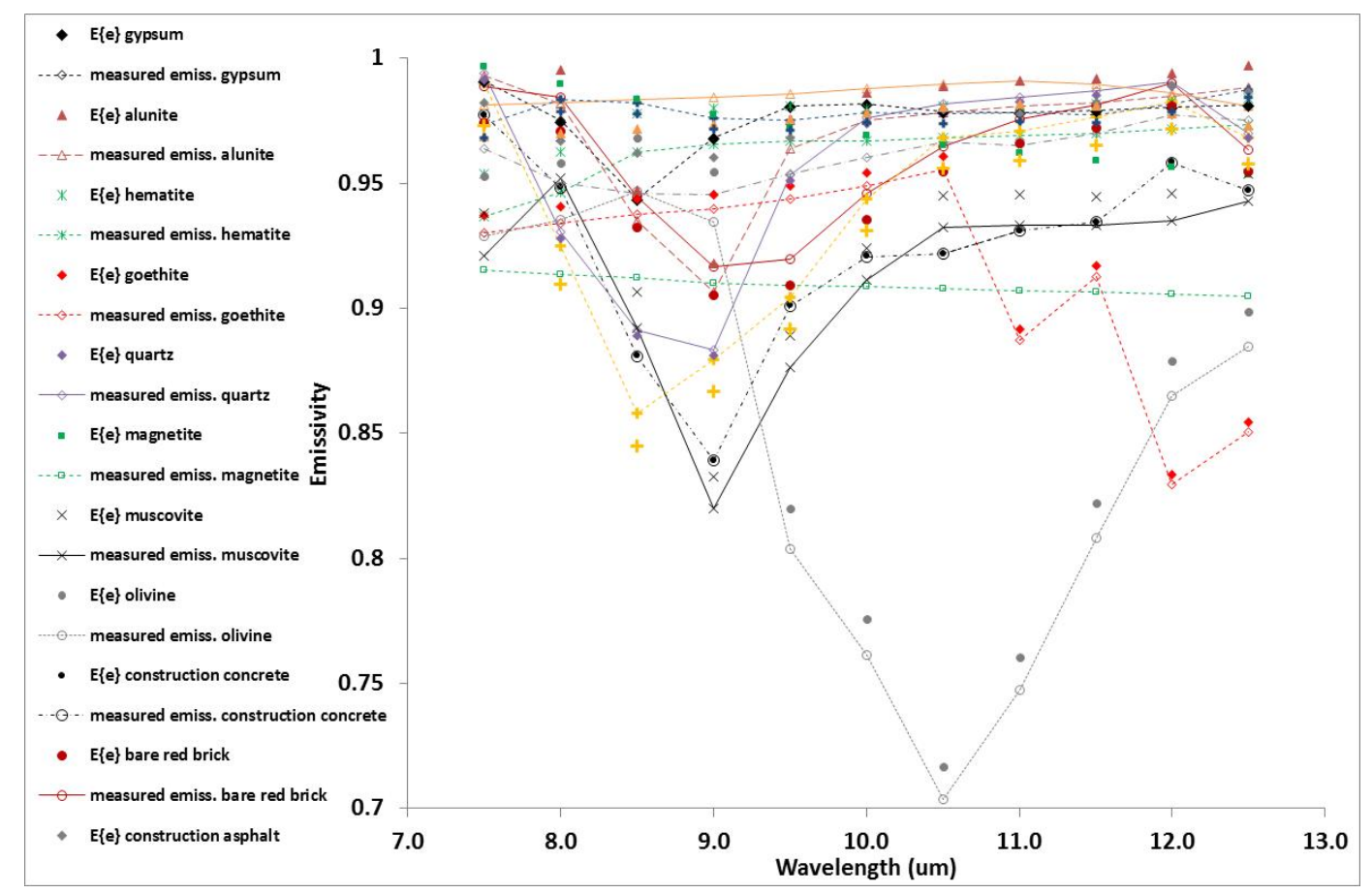

Fig. 2. Emissivity of different mineral species of interest of natural and manmade surfaces. True values are represented by lines with empty markers, the corresponding emissivity values by MaxEnTES method are represented by full marker.

The Planck functions are calculated for a temperature value of $300 \mathrm{~K}$ for each target.

The MaxEnTES algorithm reconstructs both the range and the spectral shape of the target emissivity, with the only exception of the magnetite (pointed out in the outliers of the scatter plot in figure 3 showing the retrieved emissivity values versus the true ones): the magnetite spectrum, being approximately flat, represents a mathematical difficulty in separating Plank function and emissivity, thus the retrieval fails. 


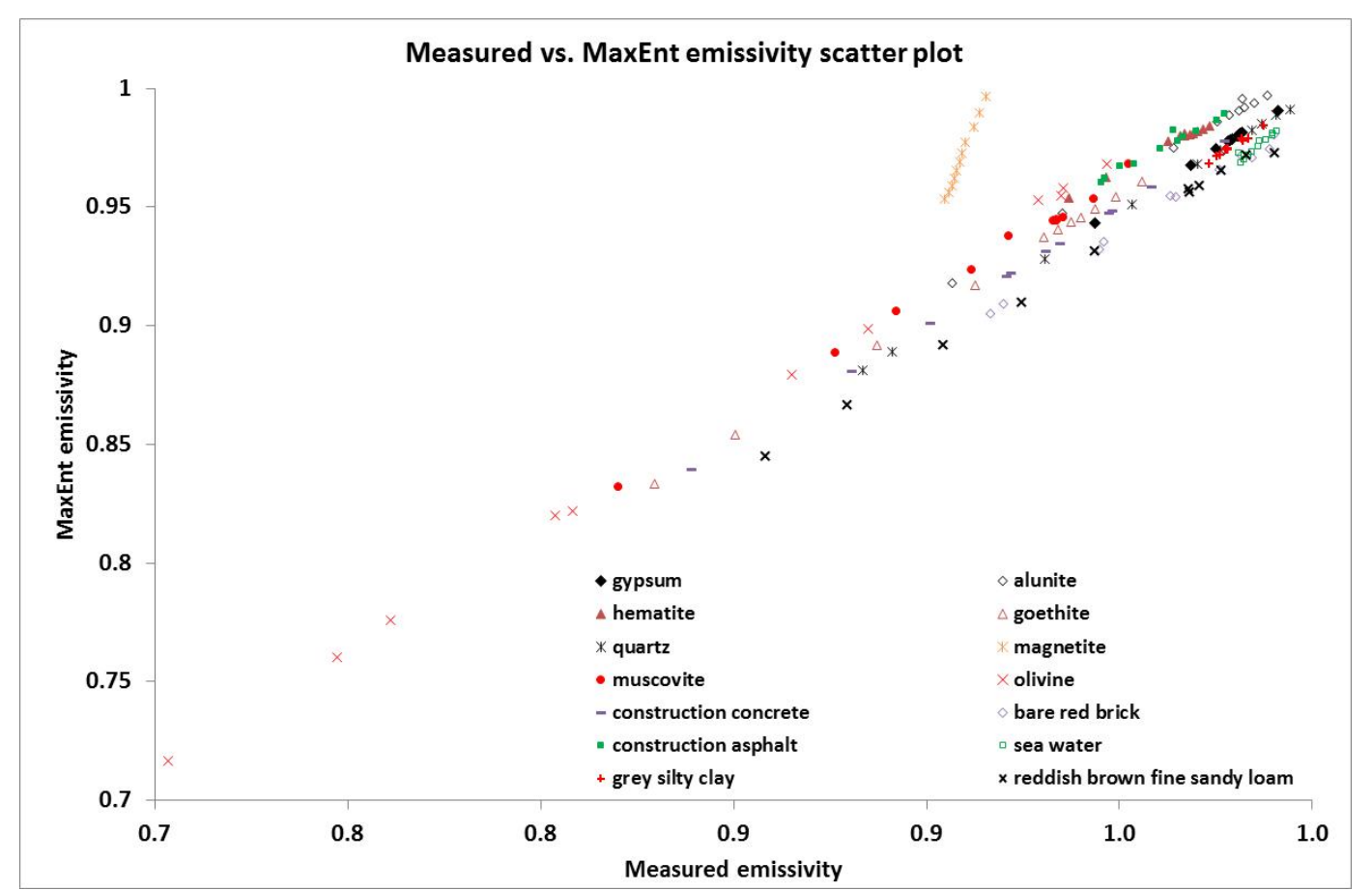

Fig 3: Scatter plot of measured (true) versus calculated values of emissivity values used in figure. 2

In figure $4 \mathrm{a}$ the relative difference (\%) of the first estimate temperature $t_{0}$ (black series), of the final estimate of the temperature (dark grey series) and maximum error on all the channels of the emissivity spectrum are represented while the uncertainty on target temperature retrieval is shown in figure $4 \mathrm{~b}$.
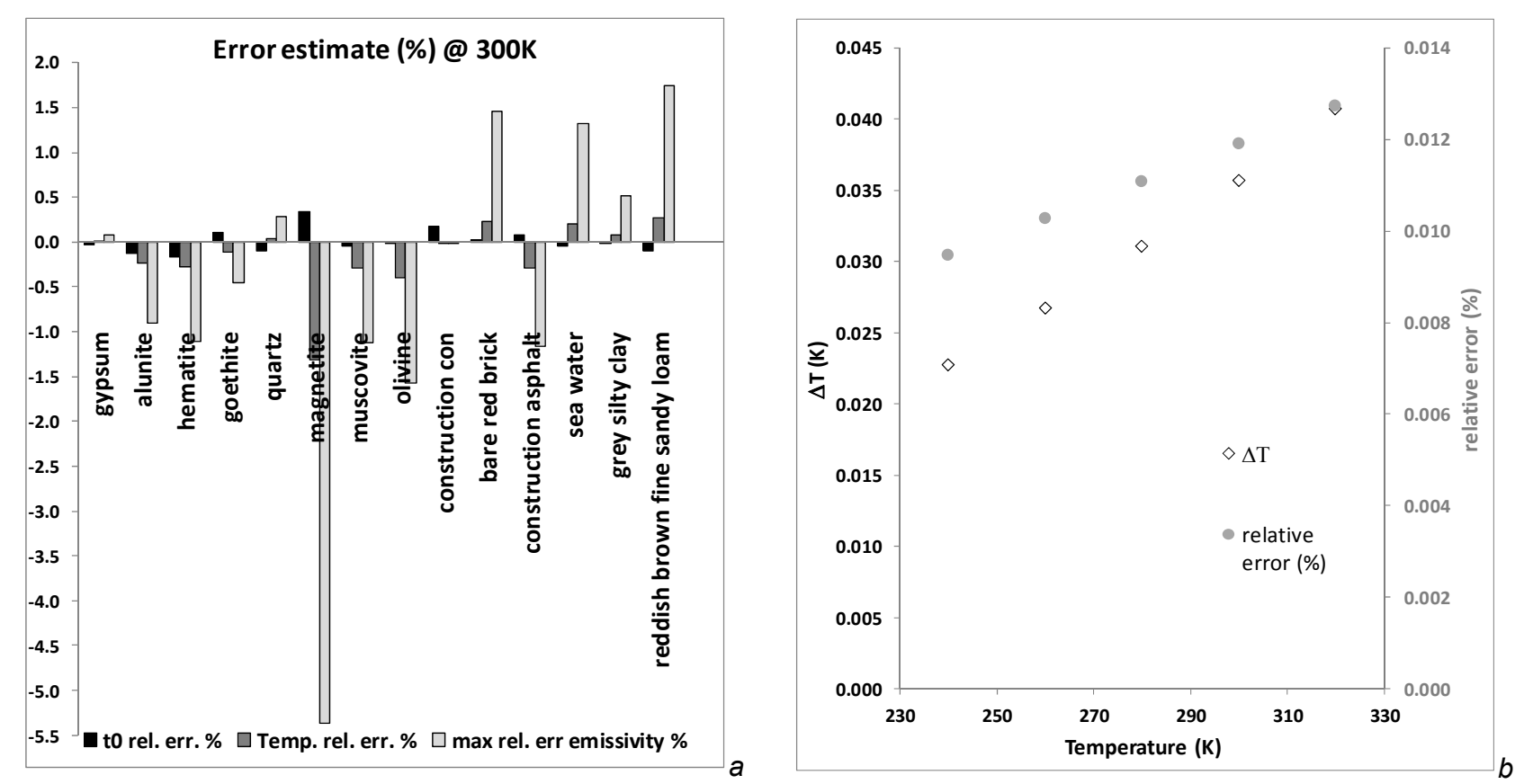

Fig. 4: (a) Relative difference (\%) of the first estimate temperature $\boldsymbol{t}_{0}$ (black series), of the final estimate of the temperature (dark grey series) and maximum error on all the channels of the emissivity spectrum. (b) Temperature difference (left vertical axis, diamond marker) and relative error (expressed in \%, right vertical axis, full round markers) between true and retrieved temperature. Gypsum emissivity spectrum at constant temperature of $300 \mathrm{~K}$ has been used.

The robustness of the MaxEnTES procedure towards noisy input has been tested by adding a random noise with uniform distribution in the radiance spectra in input. For different values of temperature a statistically relevant set $(\sim$ 
1000) of spectra has been generated. Then the MaxEnTES method has been tested on these spectra for retrieving both temperature and emissivity using sets obtained from different noise standard deviation. The result of these tests is shown in figure 5 where the relative error on temperature and emissivity as a function of the signal - noise ratio of measured radiance are provided.

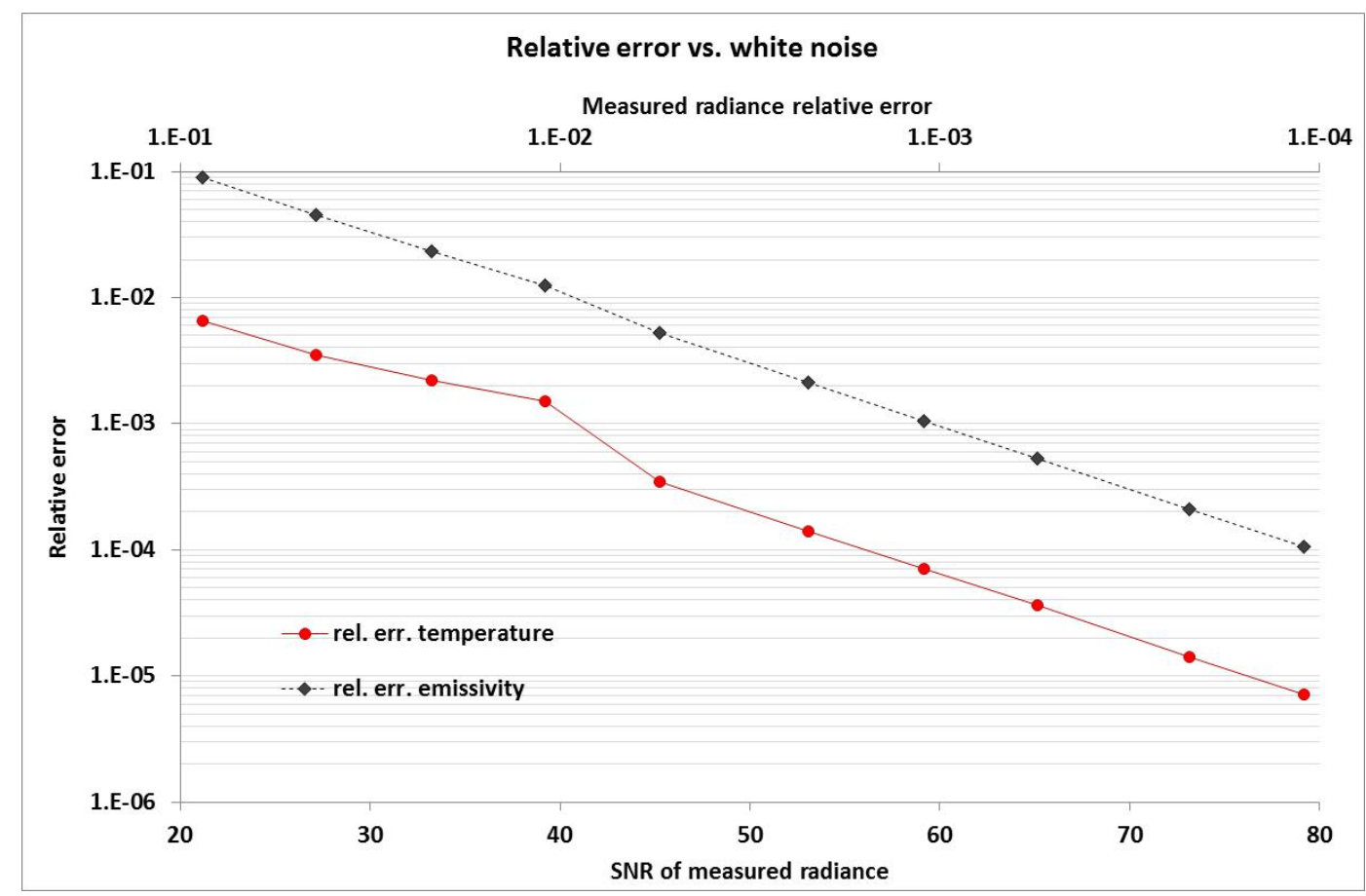

Fig. 5: Relative error on temperature (continuous line with round markers) and emissivity (segmented line with diamond markers) as a function of the signal - noise ratio of measured radiance (also the relative error is provided).

The gypsum emissivity spectrum calculated for different values of the temperature has been used as target. Random noise with uniform distribution has been added to the set of input radiances (1000 spectra for each value of the noise standard deviation).

\section{Conclusions}

The MaxEnTES procedure results accurate in reconstructing both the emissivity of the observed target and its temperature. The algorithm also shows robustness towards the noise and it is resistant to high-contrast spectral data. Moreover, its analytical formulation makes computationally fast the implementation for large amount of data (i.e. hyperspectral images).

The TRS procedure, by itself, provides an optimal first guess of the temperature, allowing the search for the maximum entropy probability distribution to converge to the correct value.

Future activities will be dedicated to the test of algorithm on natural remotely sensed data and the verification of results through an on-field validation campaign. A further improvement could be represented by the use of a non-sharp metric (i.e. a "smoothed" function instead of the one that defines the integration boundaries), allowing the weighting of the possible states of the system in a more physically realistic way.

\section{REFERENCES}

[1] Barducci A., Pippi I., "Temperature and emissivity retrieval from remotely sensed images using the "Grey Body Emissivity" method". IEEE Trans. Geosci. Remote Sens. Vol. 34, pp. 681- 695, 1996.

[2] Payan V., Royer A., "Analysis of Temperature Emissivity Separation (TES) algorithm applicability and sensitivity". Int. J. Remote Sensing, Vol. 25 (1), pp.15-37, 2004

[3] Jaynes E. T., "Prior Probabilities". IEEE Transactions on System Science and Cybernetics, sec-4 (3), pp.227$241,1968$. 
[4] Shannon C. E.; "A Mathematical Theory of Communication". Bell System Technical Journal vol. 27, pp. 379423 \& 623-656, 1948.

[5] Jaynes E. T, "Information theory and statistical mechanics". The Physical Review, vol. 106(4), pp. 620 $630,1957$.

[6] Jaynes E. T, "Information Theory and Statistical Mechanics". Statistical Physics, p. 181, K. Ford Ed., New York: Benjamin, 1963.

[7] Salisbury J. W., Walter L. S., Vergo N., and D'Aria D. M., "Infrared (2.1- 25 micrometers) Spectra of Minerals", Johns Hopkins University Press, 1991. 